\section{Percutaneous or Open Surgical Biopsy in a Case of Symptomatic Neoplastic Brachial Plexus Infiltration?}

\section{Introduction \\ $\nabla$}

Symptoms related to lesions of the peripheral nervous system (PNS) may be the initial clinical manifestation of a previously unknown tumor, arising from either the nerve tissue (e.g., schwannoma) or - more common - from non-neurogenic structures, such as lung cancer in pancoast tumors, metastatic lymph node infiltrations or lymphomas.

Percutaneous biopsy obtained from tumors presenting with symptomatic lesions of the PNS may include the risk of causing or worsening of neurological impairment and neuropathic pain due to needle trauma (Stöhr M. Nervenläsionen durch Injektion und Punktion. In: Stöhr M. Iatrogene Nervenläsionen. Georg Thieme Verlag; Stuttgart New York: 1996; Horowitz SH. Neurology 1994; 44: 962-964).

Compared to needles for injections, biopsy needles (e.g., high-speed thru-cut biopsy systems) are larger in diameter (mostly in the range of 14-18 Gauge with a core diameter of about $2 \mathrm{~mm}$ ), thus the tissue volume exposed to needle trauma is much more extended. Anatomical studies show that diameters of the trunci converging to the brachial nerve plexus (truncus sup. mean $6.7 \mathrm{~mm}$, range $4.1-$ $9 \mathrm{~mm}$, truncus med. mean $4.99 \mathrm{~mm}$, range $3.8-8 \mathrm{~mm}$ and truncus inf. mean $6.68 \mathrm{~mm}$, range $3.7-9 \mathrm{~mm}$ ) are significantly larger than core diameters of biopsy needles (Lang J. Klinische Anatomie der Halswirbelsäule. Georg Thieme Verlag; Stuttgart New York: 1991). The risk for neurological impairment is particularily high in core biopsy needle trauma when such nerves are afflicted which consist of large nerve fascicles, surrounded by small layers of epidural tissue (Selander D, Sjöstrand J. Acta anaesthesiol scand 1978; 23: 622) and it has to be assumed, that altered mechanical properties of nerves affected by neoplastic infiltration, dislocation and compression may increase the risk for core biopsy needle trauma too. Furthermore, neuropathic

\section{License terms}

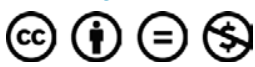

pain following nerve trauma is more common in nerves containing a high proportion of somatosensory and autonomic nerve fibers, resp., like the median and tibial nerves.

\section{Case}

$\nabla$

An 81-year-old lady with a history of myelodysplastic syndrome started to suffer from causalgia radiating from the right shoulder to the right forearm, including radial-sided wrist and fingers. She also noticed progressive weakness of her right shoulder and impaired arm movements. Physical examination showed a large mass in the right supraclavicular fossa.
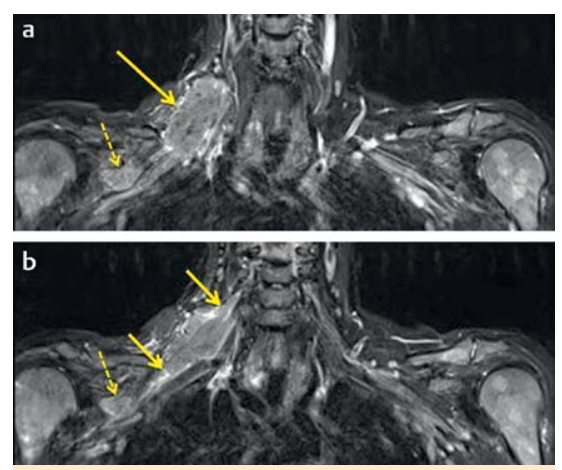

Fig. 1 a, b (MR imaging; coronal T2 with fat supression) A large supraclavicular tumor mass is clearly shown (large arrow). More posteriorly, tumor extensions towards the plexal structures are also visible (short arrows). Please note a second small tumor manifestation more laterally (dashed arrow).
On neurological examination right arm reflexes were absent and motor function of shoulder and arm muscles were both markedly reduced to $0-1$ and hand function to 4 , corresponding to the MRC scale. Sensory function was decreased at the anterior part of the shoulder and the upper arm and the radial-sided forearm, hand and thumb. Compression of the tumor resulted in burning sensations and electric dysaesthias, radiating to the right arm.

Magnetic resonance imaging (MRI) revealed a large tumor mass located in the medial supraclavicular fossa ( $\bullet$ Fig. 1a,b) in close proximity to the truncal fibres of the cervicobrachial plexus which are mainly pushed backwards ( $\bullet$ Fig. 2a,b). Noteworthy, there is also evidence of subacute denervation and subsequent atrophy of the homolateral periscapular muscles, resulting from neoplastic nerve infiltration ( $\bullet$ Fig. 3a,b).

During percutaneous biopsy performed from an anterior approach ( $\bullet$ Fig. 4 ), the same dysaesthesias occurred and subse-

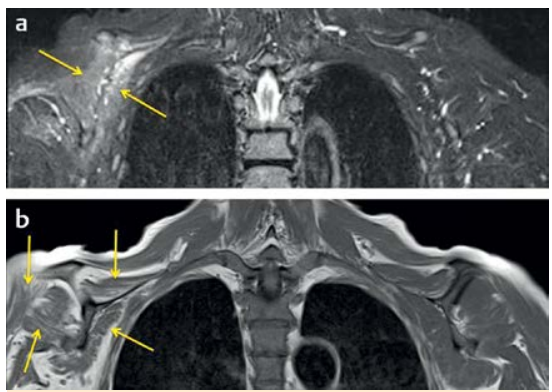

Fig. 3 a, b (MR imaging; a coronal T2 with fat suppression, $\mathbf{b}$ coronal T1) Suquelae of plexus infiltration: there is evidence of a neurogenic muscle denervation pattern of the right periscapular muscles. Please note the muscle edema reaction (arrows in a), representing subacute muscle denervation. Furthermore, there is also evidence of muscle atrophy as it is clearly depicted in T1-weighted image (arrows in $\mathbf{b}$ ). Left side of the shoulder girdle is displayed for comparison.
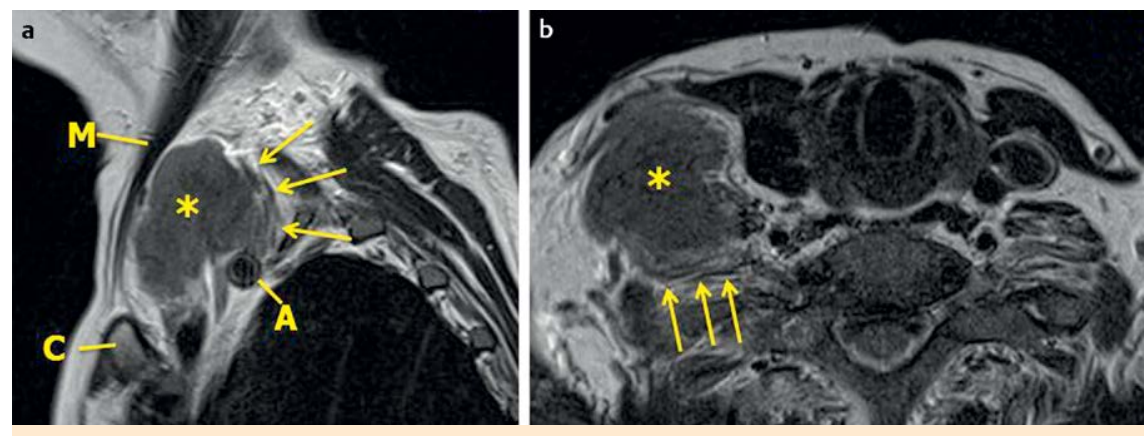

Fig. 2 a, b (MR imaging; a T2 weighted sagittal plane, b T2 weighted image axial plane) A slightly hyperintense tumor mass is shown (asterisk). Plexal fibres are pushed backwards and can be visualized around posterior aspect of the tumor (short arrows). There is no clear evidence of an intratumoral encasement of plexal structures. A subclavian artery; C clavicle; M sternocleidomastoid muscle. 


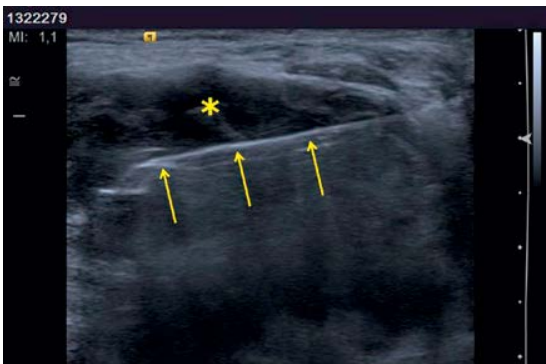

Fig. 4 Gray-scale ultrasonographic picture, obtained during the biopsy procedure. A 14-gauge high-speed thru-cut core biopsy needle (arrows) is located within the hypoechogenic tumor mass $\left({ }^{*}\right)$. No neural fibre structures could be visualized within the tumor sonographically prior to the biopsy (pre-inteventional tumoral apperance not shown). (linear ultrasound transducer, an average ultrasound frquency of $14 \mathrm{MHz}$ was applied).

quently increased weakness of the handmuscles was noted while no worsening of pain or additional sensory dysfunction could be observed. Histological work-up revealed a soft-tissue manifestation of a plasmo-blastic plasmacytoma which has been hitherto unknown.

\section{Discussion}

Large-sized tumors almost always involve neural structures, simply due to their dimensions. However, smaller tumors are capable to infiltrate, to compress or to dislocate peripheral nerves, thus leading to neurological deficit and pain.

Diagnostic MR imaging and dedicated ultrasonographic imaging are both able to visualize nerve routes involved and to identify distorted peripheral nerves. In such a scenario when histological diagnosis is urgently needed for proper treatment, planning the track for specimen sampling is of paramount importance. Similar to biopsies of malignant bone tumors, an interdisciplinary team, consist- ing of neurologists, interventional radiologists, and surgeons should evaluate the particular case. Superficial tumor location and clear identification of nerve structures involved should allow a percutaneous approach under ultrasonographic guidance. However, when both the complexity of tumor infiltration pattern and neural pathways (e.g., cervicobrachial and lumbosacral plexus) may interfere with safe needle guidance, thus, an open surgical biopsy should be preferred to avoid further nerve tissue damage.

To the best of our knowledge, there is no study available comparing both diagnostic biopsy procedures prospectively. So far evidence based recommendations concerning the invasive diagnostic process in symptomatic neoplastic brachial plexus infiltration from recent review articles are not available (Jaeckle KA. Semin Neurol 2010; 30: 254-262).

K. Pfadenhauer, T. Grieser, K. Scheglmann; Germany 